\title{
Light scattering and optical diffusion from willemite spherulites
}

\author{
Kevin M. Knowles* ${ }^{1}$, Haider Butt*2 ${ }^{2}$, Afif Batal ${ }^{2}$, Aydin Sabouri $^{2}$, Carl J. Anthony ${ }^{2}$
}

${ }^{1}$ Department of Materials Science and Metallurgy, University of Cambridge, 27 Charles Babbage Road, Cambridge, CB3 OFS, UK

${ }^{2}$ School of Mechanical Engineering, University of Birmingham, Edgbaston, Birmingham B15 $2 T T, U K$

*kmk10@cam.ac.uk, Tel: +44 1223334312

Keywords: Diffraction, Diffusers, Light scattering, Nanoscale spacings, Willemite

\begin{abstract}
Willemite is a zinc silicate mineral used in modern day pottery as a decorative feature within glazes. It is produced by controlled heat treatment of zinc oxide-containing ceramic glazes. The heat-treated glazes devitrify, producing thin nanoscale needle-like willemite crystals growing in spherulitic morphologies through branching of the needles. We show here that this resulting morphology of willemite crystals in an inorganic glass matrix has a previously unreported strong interaction with light, displaying remarkable optical diffraction patterns. Thin sections of such spherulites act as optical diffusers, enabling light beams to be spread up to $160^{\circ}$ in width. Analysis of the interaction between the willemite spherulites and light suggests that the high density of willemite crystals in the spherulites and the length scales associated with both the thickness of the needles and the spacings between branches are together responsible for this optical diffusion behaviour.
\end{abstract}

\section{Introduction}

Willemite, $\mathrm{Zn}_{2} \mathrm{SiO}_{4}$, also known as zinc orthosilicate, is the main constituent of crystalline glazes seen in modern day pottery [1]. Various crystalline glazes have been produced for hundreds of years by careful heat treatment in order to decorate pots, plates and other ceramic products [2]. Willemite is striking in that it produces macrocrystals in the form of spherulites which can be $150 \mathrm{~mm}$ or more in diameter [3,4]. As a mineral, it is most notably associated with fluorescent crystals from Franklin in New Jersey [5]. Indeed, synthetically produced willemite doped with either manganese or beryllium was used for the first generation of phosphors in fluorescent tubes [6-11]. Recent research on nanophosphors has reignited interest in using suitably doped willemite in nanocrystalline form embedded in a host matrix for various optical and biological applications [12]. 
Prompted by our recent work on devitrite, in which we have shown that needle-like crystals of devitrite embedded in a silica-rich glass matrix enable laser light to be diffused to large scattering angles [13], we have examined in the work reported here the potential for willemite spherulites also to act as optical diffusers. Optical diffusers are devices that spread out or scatter focused light to give soft light. They are widely used in the optical industry. For example, the use of diffused reflectors can improve and reduce the cost of photovoltaic systems [14]. Optical diffusers are also used in camera flash lights. For photothermal therapies, diffusing microneedles are used to deliver and spread thermal energy [15]. In addition, diffusing screens are needed to increase the field of view and control the light intensity of display devices [16].

The efficiency of an optical diffuser is characterised by its ability to transmit the entirety of the incident light and the homogeneity of its diffused profile [17]. Optical diffusers can spread concentrated light in one or two dimensional diffusion patterns with varying beam widths. These diffusers are commercially produced using expensive and complicated nanofabrication manufacturing processes, such as photolithography to create 3D nanostructures which diffuse light [18-20].

In common with needles of devitrite [13], the willemite samples examined in this paper are also characterised by needle-like crystals embedded in a silica-rich glaze originating from a common centre and spanning outwards $[1,3,4]$. Importantly, we show here that the widths of these willemite needles and the spacings between these needles of willemite are of the order of the wavelength of visible light and comparable with those seen in devitrite spherulites [13]. As a consequence, it is entirely reasonable to anticipate that this arrangement of willemite needles can also be used to diffuse incident, focused light; this is indeed the case. We are therefore able to report here for the first time optical experiments from thin $30 \mu \mathrm{m}$ sections of willemite spherulites embedded in a silica-rich glass matrix which show the remarkable way in which light transmitted through these thin sections can be diffused to high angles. The reproducibility of the observations clearly depends on the repeatability of the fabrication process, but it is evident that thin sections of similar arrangements of needleshaped crystals of willemite in similar spherulites will show very similar optical diffraction effects.

The phenomenon of the scattering of light by small particles and the practical consequences of this scattering is familiar in a number of scientific fields [21,22]. For the work we describe here, the particles are groups of needles, rather than a single homogeneous sphere of one material embedded within another material, for which the analytical Mie solution is appropriate [23]. Clearly, depending on the density of needles in the inorganic glass matrix, the willemite spherulites can be envisaged either as needles of willemite embedded in a glass matrix or as clusters of needles surrounded by residual glass. The relative proportions of the willemite and glass, the refractive index difference(s) between willemite and glass and the scale of the spherulite morphology are all key considerations in appreciating how the spherulites scatter light.

Willemite is an optical positive trigonal crystal, the principal refractive indices of which are $n_{\mathrm{O}}=1.6893$ and $n_{\mathrm{E}}=1.7179$ at the wavelength of sodium $D$ light [24] for the ordinary ray and extraordinary ray respectively. It has a trigonal crystal structure belonging to the $R \overline{3}$ 
space group with $a=13.948 \AA$ and $c=9.315 \AA$ using hexagonal indices to define the unit cell dimensions [25]. By comparison with devitrite, the principal refractive indices of which are $1.565,1.570$ and 1.580 [26], willemite has a significantly higher mean refractive index than ordinary soda-lime-silica glass, the refractive index of which is 1.52 [27]. Hence, for similar morphologies and similar length scales for needles within a silica-rich matrix of a refractive index $\approx 1.52$, it might be expected qualitatively that willemite has a greater potential for light scattering than devitrite. For example, in Rayleigh-Gans scattering [21,22], in which the particles can be regarded as small non-interacting dipole scatterers [22], the scattered intensity scales as

$$
|m-1|^{2}
$$

for light incident on an isotropic sphere of refractive index $m_{1}$ embedded in an isotropic matrix of refractive index $m_{2}$, and where $m=m_{1 /} m_{2}$.

When producing commercial crystalline glazes, the glaze is applied to a ceramic body by first preparing an aqueous slurry of the dry components of the glaze recipe. A number of methods can be used to transfer the glaze ingredients to the ceramic body, such as dipping, brushing, pouring or spraying $[3,4]$. A noticeable feature of the heat treatment process to produce crystalline glazes is that at the high temperatures required to melt the glaze ingredients, typically at $1280{ }^{\circ} \mathrm{C}$ (i.e., cone 9 heated at $150{ }^{\circ} \mathrm{C} \mathrm{hr}{ }^{-1}$ ) or $1305{ }^{\circ} \mathrm{C}$ (i.e., cone 10 heated at $150^{\circ} \mathrm{C} \mathrm{hr}^{-1}$ ) [3,4], the glaze is very fluid. Growth of the willemite spherulites is then usually undertaken at temperatures around $1100{ }^{\circ} \mathrm{C}[3,4]$. It is common for glazed ceramic wares to be held within the crystal growth temperature regime for $3-6 \mathrm{hrs}[3,4]$. The spherulites arise as a consequence of heterogeneous nucleation and the subsequent crystal growth of the willemite crystals in spherulitic form [28] - the temperatures and times chosen are a practical compromise between the ease of nucleation and the rate of crystal growth to produce spherulites of the desired size and aesthetic appearance [3,4].

Although there are clear technological challenges to overcome, one of which is the slow flow of the glass at the temperatures used for efficient crystal growth of the willemite, we envisage that technology for producing bulk inorganic glass can be adapted to produce crystalline glazes either as bulk inorganic glass or as thin sheets of inorganic glass. This glass could then be suitably heat treated to produce willemite and subsequently cut to size for a final commercial product as an optical diffuser. Just as with devitrite grown within soda-lime-silica glass [13], the advantage that such a diffuser would have over conventional diffusers would be its resistance to temperatures up to the glass transition temperature of the silica-rich glaze, i.e., to over $500{ }^{\circ} \mathrm{C}$.

\section{Experimental}

Specimen preparation procedures for transmitted light polarised light microscopy and scanning electron microscopy (SEM) observations of the spherulites from pots have been described by Knowles and Freeman [1]. Fast Fourier Transforms (FFTs) [29] of digital images produced in the transmitted light polarised light microscope of regions used for optical diffusion experiments were generated to help quantify the relationship between the 
microstructure of the spherulites and their optical diffusion behaviour seen experimentally. The far field diffraction pattern simulations produced using this FFT procedure are based on the Fraunhofer diffraction principle [30-32].

Samples for transmission electron microscopy (TEM) to examine the microstructure of the willemite spherulites at a resolution below that of visible light were prepared by first using the procedures to make samples thin enough for transmitted light microscopy. Selected regions from these samples containing spherulites were dimpled, mounted on to $3 \mathrm{~mm}$ diameter copper rings and ion beam thinned to electron beam transparency using argon ions. TEM was undertaken in a JEOL 200CX transmission electron microscope operating at $200 \mathrm{kV}$. A heavy carbon coating was necessary on the TEM specimens to prevent them charging while under observation in the transmission electron microscope.

A schematic diagram of the experimental arrangement for initial optical experiments performed to examine the ability of these assemblies of thin willemite crystals to diffuse light is shown in Fig. 1. This is similar to the experimental arrangement used for our previous study on the ability of devitrite to diffuse light [13]. The samples were mounted horizontally directly above a base which held a laser light source pointing normally to the sample. A hemispherical screen was placed above the sample so that the base of the screen was parallel to the plane of the sample and concentric with both the laser pointer and the willemite sample. The screen had a radius of some $15 \mathrm{~cm}$, giving enough distance to be able to view the far-field diffraction patterns. The sample was then illuminated with a $633 \mathrm{~nm}$ wavelength red laser source which was transmitted through the crystals and diffracted onto the hemispherical screen. The profile of the transmitted beam was then captured by a camera.

For more detailed optical experiments, the exact angular distribution of light diffused by the willemite sample was determined by mounting the sample on a vertical post on a base which was able to rotate, as shown in Fig. 2. The laser pointer was then mounted on a second post on this base immediately behind the willemite sample, so that it was pointing normal to the surface of the willemite sample. This rotatable base was linked to an electric motor. As a result, the sample and the laser pointer were always in the same position relative to each other and were able to rotate simultaneously. A light power meter was mounted on a third fixed post almost $10 \mathrm{~cm}$ away from the laser pointer source. This enabled the far-field light intensity to be measured as a function of angle from the sample normal direction.

A cylindrical tube was fixed in front of the light power meter to ensure that no stray light was measured. It was important to make sure that the alignment of the laser was perfect relative to the light power meter, so that within experimental error the laser was pointing directly into the power meter when an angle of $0^{\circ}$ was set for the rotatable base. A computer was used both to control the electric motor rotating the laser and the sample and to store the readings from the light power meter at $1^{\circ}$ steps of rotation.

Finally, further scattering measurements were also performed with a beam of white light. The white light beam used in these experiments was larger in diameter than the regions of willemite crystals, as a consequence of which a significant level of undiffracted light was observed in the experiments. As with the experimental work using $633 \mathrm{~nm}$ wavelength red laser light, the sample was mounted on a glass slide and light was shone through the slide to examine the scattering profile. 


\section{Results}

\subsection{Microstructural observations}

The samples considered in this work were thin sections of spherulites extracted from a pot purchased at a market stall in Barcelona, believed to have originated from the Catalunya region of Spain [1]. A low magnification reflected light micrograph of a group of spherulites from this pot is shown in Fig. 3. The glaze is 100-200 $\mu \mathrm{m}$ thick, and so these spherulites are predominantly two-dimensional. Compositional analysis of this glaze in previous work confirmed that the coloration of the glaze and the crystals was due to the incorporation of cobalt in the glass frit used for the glaze [1]. Cobalt ions segregate preferentially to the willemite crystals during heat treatment because $\mathrm{Co}^{2+}$ ions have a preference for tetrahedral coordination and can substitute for the tetrahedrally coordinated $\mathrm{Zn}^{2+}$ ions in willemite [1]. The periphery of these spherulites is lighter in colour than the main body of the spherulites and will have been produced at the end of the heat treatment period, either through natural cooling after the furnace has been switched off, or through a deliberate and steady lowering of the temperature of the furnace used for the growth of the spherulites [1].

Closer examination of spherulites by polarised light microscopy of thin sections of the crystalline glaze using a sensitive tint inserted at $45^{\circ}$ to the extinction directions showed the fibrous nature of the individual willemite crystals within the spherulites, such as in the example shown in Fig. 4, with the fibre direction [0001]. This also showed that, at the periphery of the spherulites, the fine nature of the crystals could not be distinguished by transmitted light polarised light microscopy, indicating that the crystals comprising the periphery were of sub-micron or nanoscale dimensions. There was evidence of branching of the individual willemite crystals in the main body of the spherulites immediately adjacent to this nanocrystalline region, but it is apparent from Fig. 4 that optical microscopy suffers from clear resolution limitations when attempting to study the fine detail of this branching.

SEM observations of samples, thinned sufficiently so that individual groups of crystals could be identified by polarised light microscopy immediately prior to their selection for dimpling and further thinning for TEM investigations, showed the branching behaviour with greater clarity. Examples are shown in the two secondary electron micrographs in Fig. 5. The contrast in this microscopy mode highlights the zinc-containing willemite crystals as long, thin, white branched needles on a darker grey background of silica-rich glass of a lower mean atomic number than the willemite crystals. It is also important to appreciate that SEM observations such as those in Fig. 5 probe the first micrometre or so of the specimen under examination, which is why both the willemite needles and the glass matrix within the spherulites are evident. By contrast, the samples for transmitted light microscopy were some $30 \mu \mathrm{m}$ thick, so that the overlap in projection of willemite needles in Fig. 4 in the region labelled 'Primary willemite needles' has the effect of masking any evidence for glass within the spherulites.

Both examples in Fig. 5 show the dendritic nature of the branching of the willemite crystals, and, in the case of Fig 5(b), towards the top of the micrograph, the fine scale nature 
of the willemite crystals in the adjacent periphery region surrounding the larger branched willemite crystals in the main body of the spherulite. The impression gained from such observations is of crystals generally growing along the willemite [0001] direction, but able to branch easily along directions away from [0001]. Direct measurements taken from the micrograph in Fig. 5(a) would appear to suggest that the [0001] growth direction bisects an angle between two branches of $32-36^{\circ}$, whereas the same angle measured in Fig. 5(b) takes a range of values between $22^{\circ}$ and $45^{\circ}$, depending on the region chosen. Such variations in measurements highlight the effect of projectional geometry on angle measurement using SEM - the true angle between equivalent branch directions will be greater than, or equal to, that seen by direct measurement from an SEM micrograph.

Further information on the crystallization and branching behaviour was obtained from TEM observations, such in the examples shown in Fig. 6. Groupings of willemite crystals, all with the same electron diffraction pattern, could be readily found in regions where the size of the crystals indicated that they were either from within the periphery region, or very near to it, while just within the main body of the spherulite. The willemite crystals in Fig. 6(a) were imaged with the electron beam parallel to the [ $\overline{1} 2 \overline{1} 0]$ willemite direction ([010] in the 3 index notation). The dominant [0001] direction is marked on this micrograph. The angle measured between [0001] and each of the two clearly visible branches was $17^{\circ}$, so that the two branches make an angle of $34^{\circ}$ with one another in this micrograph. Fine branching within the periphery region is also evident in Fig. 6(b), taken with the electron beam parallel to the $[10 \overline{1} 0]$ willemite direction ([210] in the 3-index notation). Branching behaviour with a measured a branch angle of $17^{\circ}$ is also seen in Fig. 6(c), even though in this micrograph the [0001] growth direction does not lie within the plane of the micrograph - the electron beam was parallel to the [ $\overline{1} 101]$ willemite direction ([ $\overline{1} 11]$ in the 3 -index notation).

Away from the spherulites, and in glassy pockets within the spherulites, there was evidence of phase separation within the glass on a scale of $100 \mathrm{~nm}$, such as in the example shown in Fig. 6(d). The interconnectivity of the phase separation in this micrograph is consistent with spinodal decomposition, and is of the same scale as the spinodal decomposition phase separation seen in other inorganic glass systems [33,34]. Additional contrast in the darker regions arises from devitrified zinc-rich areas within the phaseseparated glass. This phase-separating nature of the glaze as part of the process leading to the formation of crystalline phases within the glaze agrees with observations reported by others both for crystalline glazes [35] and Ru ware glaze [36].

Thus, overall, it is evident from these observations that the microstructure of the essentially two-dimensional spherulites can be described as branched nanoscale needles of willemite embedded in a glassy matrix. The density of the needles per unit area within the spherulites is such that the spacings between the needles are of similar length scales to the diameter of the needles. The phase separation within the glass is on a much finer length scale of $\approx 100 \mathrm{~nm}$. Within the primary nucleated willemite spherulites, the widths of the long needles can be slightly greater than the wavelength of light, but it is evident from the difficulty in resolving unambiguously single needles within groupings of needles in the transmitted light microscope that the scale of the microstructure as a whole is similar to the scale of wavelengths within visible light. 


\subsection{Optical experiments}

An example of a profile of the transmitted beam through a $30 \mu \mathrm{m}$ thick section of willemite spherulite chosen at random is shown in Fig. 7. The beam diameter of the laser beam used was $1 \mathrm{~mm}$, so that for this and other transmitted beam profiles, a relatively large region dense in primary willemite needles within thin sections of spherulites was sampled by the laser beam. Qualitatively, it is evident from this image that the diffusion profile is able to extend to large angles, in fact almost $80^{\circ}$ from the normal along one line of longitude, in comparison to $10-15^{\circ}$ on the line of longitude perpendicular to it.

Different arrangements of the crystals produced different optical diffusion patterns. To quantify the relation between the arrangements of the willemite needles and the diffusion patterns, simulations were performed to find a match with experiments. Examples of three distinct regions within the spherulites are shown in the polarised light images in Figs. 8 (a)-(c). In these images the extinction directions are vertical and horizontal. The image in Figure 8(a) is of the centre of a spherulite, the nucleation site appearing to be a dust particle acting as a heterogeneous nucleation site for the nucleation and subsequent growth of the willemite needles.

It is apparent from the FFTs of these images shown in Figs. 8 (d)-(f) that, as expected, the arrangements of the willemite needles dictate the far-field diffraction pattern, so that light is diffused in a direction orthogonal to the willemite needle axes. Lines extending horizontally and vertically from the centre of each of these simulations arise from the limitations of the FFT software. The trends seen in these simulations are consistent with experimental measurements of optical diffusion from these three regions shown in Figs. 8 (g)-(i).

These results confirm that if the primary willemite crystals within a spherulite are arranged in a certain pattern or array, the main features in the overall optical diffusion that arises when laser light is shone through the crystals can be understood qualitatively. Therefore, with experience, different diffusion patterns can be achieved by arranging the crystals in a particular manner. For example, a simple diffuse cross pattern extending to high angles can be achieved by overlapping different thin, relatively unidirectional, samples of needles of willemite, such as those in Figs 8(b) and 8(c) from regions reasonably far from the nucleation site of the spherulites. Hence, by careful choice of how this is undertaken in practice, there is the ability to create a range of diffuse beam profiles.

Details of the angular intensity profiles for two similar optical diffusion patterns through thin $30 \mu \mathrm{m}$ sections of willemite spherulites are shown in Fig. 9. In both cases, it can be seen that the willemite crystals distribute the light up to angles of $80^{\circ}$ from the incident beam direction; this is very impressive. The other important feature is that no significant peak was observed at $0^{\circ}$ (zero order) in the intensity distribution measurements, but rather a smooth symmetrical distribution about $0^{\circ}$ within experimental error. This shows that the diffuser is diffusing the majority of the incident light to high angles so that very little undiffracted light remains. Just as for devitrite [13], these data confirm that the incident light undergoes a phase modulation due to the presence of the needles of willemite. 
Further diffraction measurements were also performed with a beam of white light, the results of which are shown in Fig. 10. It is apparent that the white light is also diffused by the willemite crystals uniformly to large angles. Angular measurements shown in Fig. 10(c) confirm that the white light diffuses to angles as high as $80^{\circ}$. Optical transmission through the crystals was also studied using a transmission optical microscope connected to a spectrometer. Examples of transmission spectra and the type of crystal regions tested are shown in Fig. 10(e). The spectra from different regions had similar general profiles but varying average transmission intensities in the range 40-60\%. In these experiments, only a proportion of the scattered light was collected by the microscope objective; this leads to lower transmission readings than the actual degree of transmission.

\section{Discussion}

The results in Section 3 show that the willemite crystals within the spherulites have a strong interaction with visible light, with scattering in the forward direction of a light beam to high angles. Examination of the microstructure within the spherulites shows a range of length scales present which are all relevant to an understanding of why the scattering is so noticeable.

It is evident from Fig. 8 that when groups of thin branched needles are arranged in a quasi-parallel arrangement, optical diffusion is most efficient perpendicular to the quasiparallel axis, consistent with what would be expected from a very simple picture of the microstructure as a periodic grating. On the basis of such a very simple picture, the maximum diffraction angle produced for an incident beam of light going through a periodic grating normal to the beam can be easily obtained using the equation

$$
\lambda=d \sin \theta
$$

where $\lambda$ is the wavelength of incident light, $d$ is the spacing between the elements of the grating, and $\theta$ is the angle between the incident beam and the scattered beam [32]. For $\theta=80^{\circ}$ and a wavelength $\lambda=633 \mathrm{~nm}$, such a periodic grating would require $d=643 \mathrm{~nm}$. Of course, this is far too simple a picture of the microstructure, but it is useful as a reminder that length scales of the order of light are required in a microstructure to be able to scatter light.

A more considered approach is to examine the mathematical physics of light scattering and extract useful results from known scientific problems within this field. In this context, the scattering of light by independently scattering non-absorbing homogeneous spheres is a problem with a known analytical solution [21-23,31]. Following the approach of van de Hulst [21], for willemite embedded in a medium of inorganic glass, it is useful to define a relative refractive index $m=m_{1} / m_{2}$, where $m_{1}$ is a mean refractive index for willemite and $m_{2}$ is the mean refractive index of the glass. Taking $m_{1}=\left(2 n_{\mathrm{O}}+n_{\mathrm{E}}\right) / 3$ and $m_{2}=1.52$ gives $m=$ 1.12. For a spherical particle of radius $a$ and a wavelength $\lambda_{\text {vac }}$ in vacuum, a relevant dimensionless parameter, $x$, is then defined as $x=2 \pi a / \lambda=2 \pi a m_{2} / \lambda_{\text {vac }}$, where $\lambda$ is the wavelength in the medium of refractive index $m_{2}$. The problem of the scattering of light by willemite spherulites can then be reduced to an equivalent problem in the $m-x$ domain for scattering by non-absorbing spheres ([21], Ch. 10). 
Since $m$ is close to 1 , the Rayleigh-Gans scattering regime is of particular interest. For this regime to be directly relevant, the conditions

$$
|m-1|<<1
$$

and

$$
2 x|m-1|<<1
$$

pertain, so that each separate volume element of the scattering particle behaves as a Rayleigh scatterer, while interference from the waves scattered by each volume element of the scattering particle interfere because of their different positions in space [21]. The intensity scattered by a sphere under these conditions is such that as $x$ increases from a value $<<1$, the scattering changes from that of Rayleigh scattering, where there is a significant amount of backscattering, to a concentration of light towards smaller scattering angles, with a series of minima in the scattered intensity as a function of scattering angle $\theta$ [21].

If, under these circumstances, we interpret the intensity distributions seen in Figs. 7-9 in terms of equivalent sphere radii defined by the first zeroes in the scattered intensity, a first minimum arises when

$$
\frac{4 \pi a}{\lambda} \sin \frac{1}{2} \theta=4.49
$$

using $\$ 7.21$ and Table 6 of [21]. For $\theta=80^{\circ}$, this implies that

$$
\frac{4 \pi a}{\lambda}=6.99
$$

so that Eq. (4) is approaching the limit of its validity. For $\lambda=633 / 1.52 \mathrm{~nm}$, this produces a value of $a$ of $230 \mathrm{~nm}$, comparable with the Abbe resolution limit of the polarised light microscope. This is consistent with needle radii seen by TEM and the difficulty in resolving unambiguously individual willemite needles in the polarised light microscope.

For $\theta=12.5^{\circ}$ on the line of longitude perpendicular to this wide scattering distribution,

$$
\frac{4 \pi a}{\lambda}=40
$$

and so although this produces an equivalent sphere radius, $a$, of $1300 \mathrm{~nm}$, this should in principle be regarded as less reliable an estimate because the condition $2 x|m-1|<<1$ required for the Rayleigh-Gans theory to be valid no longer holds. However, despite this obvious restriction, this equivalent sphere radius highlights that the groupings of branched needles have an effective size for scattering of the order of the distances between branching nodes, rather than the lengths of the main trunks of the branched needles.

Comparing the predictions from Rayleigh-Gans scattering with more accurate full-form Mie solution predictions for scattering from homogeneous spheres embedded in a medium of differing refractive index made using the open source program MiePlot [37] shows that the predictions from the Rayleigh-Gans scattering mode are more than adequate for our purposes. Examples of predicted total intensity as a function of scattering angle for $m_{1}=1.699, m_{2}=$ $1.52, \lambda_{\text {vac }}=633 \mathrm{~nm}$ and particle sizes of $230 \mathrm{~nm}$ and $1300 \mathrm{~nm}$ are shown in Fig. 11(a) and (b) 
respectively from the full-form Mie solution for non-absorbing spheres. Qualitatively, these are remarkably consistent with the form of the experimental results seen in Figs. 7-9.

In practice of course, the degree of scattering exhibited by the willemite spherulites is a function of the density of willemite crystals per unit area in the plane perpendicular to the light source. There will also be a degree of absorption. However, we can conclude that the efficiency of the scattering is as high as we have found experimentally because of the efficient way in which willemite spherulites grow outwards within the crystalline glaze.

Finally, as we have already noted in Section 3, if the primary willemite crystals within a spherulite are arranged in a certain pattern or array, the main features in the overall optical diffusion that arises when laser light is shone through the crystals can be fully appreciated in terms of the scale of the microstructure and the arrangement of the crystals. Clearly, thin sections of similar arrangements of needle-shaped crystals of willemite in similar spherulites will show very similar optical diffraction effects. Therefore, suitably prepared thin sections from other crystalline glaze ware will be able to reproduce results very similar to the ones we have shown here.

To be able to adapt crystalline glaze technology for optical diffusers, it would be necessary to produce heterogeneous nucleation of willemite homogeneously throughout a suitable zinc-rich silicate glass, either in bulk form or as thin sheets of glass. The Portmeirion Starfire collection, in which titanium oxide was used as a suitable heterogeneously nucleating agent [1], just as in glass-ceramics [33], is an example of how heterogeneous nucleation of willemite can be achieved homogeneously throughout a crystalline glaze. In contrast to this, producers of crystalline glaze ware normally aim to produce relatively few spherulites (or 'macro-crystals' [3,4]).

\section{Conclusions}

The experiments reported here demonstrate for the first time the previously unreported observation that light scattering to remarkably high angles of up to $80^{\circ}$ can be produced from thin $30 \mu \mathrm{m}$ sections of spherulites of willemite $\mathrm{Zn}_{2} \mathrm{SiO}_{4}$ produced in crystalline glazes, in which branched needles of willemite are parallel to the plane of the thin sections. Examination of the microstructure of the willemite spherulites and a consideration of the physics of light scattering shows that the length scales occurring naturally within the spherulites are consistent with length scales required to cause this optical diffusion. As a consequence, there is potential for spherulites of willemite to be used as optical diffusers able to withstand temperatures up to $500{ }^{\circ} \mathrm{C}$. 


\section{References}

[1] K.M. Knowles, F.S.H.B. Freeman, Microscopy and microanalysis of crystalline glazes, J. Microscopy 215 (2004) 257-270.

[2] D.H. Sun, L.A. Orlova, N.Y. Mikhailenko, Types and compositions of crystalline glazes (a review), Glass and Ceramics 56 (1999) 177-180.

[3] P. Ilsley, Macro-Crystalline Glazes: The Challenge of Crystals; The Crowood Press, Marlborough, U.K., 1999.

[4] D. Creber, Crystalline Glazes, second ed., A \& C Black, London, U.K., 2005.

[5] F.H. Pough, Occurrence of willemite, Am. Miner. 26 (1941) 92-102.

[6] W.B. Nottingham, Potential and luminescence of insulated willemite cathode-ray screens, Phys. Rev. 51 (1937) 591.

[7] W.B. Nottingham, Electrical and luminescent properties of willemite under electron bombardment. J. Appl. Phys. 8 (1937) 762-778.

[8] G.R. Fonda, Phosphorescence of zinc silicate phosphors, J. Appl. Phys. 10 (1939) 408-420.

[9] E.G. Ramberg, G.A. Morton, Variation of light output with current density and classification of willemite phosphors, Phys. Rev. 55 (1939) 409.

[10] R. Kane, H. Sell, Revolution in Lamps: A Chronicle of 50 Years of Progress, second ed; Fairmont Press: Lilburn, Georgia, U.S.A., 2001.

[11] T.E. Warner, Synthesis, Properties and Mineralogy of Important Inorganic Materials, John Wiley and Sons Ltd., Chichester, U.K., 2011, Ch. 14.

[12] J. El Ghoul, K. Omri, S.A. Gómez-Lopera, L. El Mir, Sol-gel synthesis, structural and luminescence properties of MT-doped $\mathrm{SiO}_{2} / \mathrm{Zn}_{2} \mathrm{SiO}_{4}$ nanocomposites, Optical Materials 36 (2014) 1034-1039.

[13] H. Butt, K.M. Knowles, Y. Montelungo, G.A.J. Amaratunga, T.D. Wilkinson, Devitrite-based optical diffusers, ACS Nano 8 (2014) 2929-2935.

[14] J.M. Kim, P.S. Dutta, Optical efficiency-concentration ratio trade-off for a flat panel photovoltaic system with diffuser type concentrator, Sol. Energy Mater. Sol. Cells 103 (2012) 35-40.

[15] M.A. Kosoglu, R.L. Hood, J.H. Rossmeisl, D.C. Grant, Y. Xu, J.L. Robertson, M.N. Rylander, C.G. Rylander, Fiberoptic microneedles: novel optical diffusers for interstitial delivery of therapeutic light, Lasers Surg. Med. 43 (2011) 914-920.

[16] G.M. Morris, T.R.M. Sales, Structured screens for controlled spreading of light, U.S. Patent 7,033,736 B2, 2006.

[17] R. Bitterli, T. Scharf, H.-P. Herzig, W. Noell, N. de Rooij, A. Bich, S. Roth, K.J. Weible, R. Voelkel, M. Zimmermann and M. Schmidt, Fabrication and characterization of linear diffusers based on concave micro lens arrays, Opt. Express 18 (2010) 14251-14261.

[18] M. Christophersen, B.F. Phlips, Gray-tone lithography using an optical diffuser and a contact aligner, Appl. Phys. Lett. 92 (2008) 194102-1-3.

[19] M. Christophersen, B. Phlips, Gray-tone lithography using optical diffusers, U.S. Patent 8,372,578 B2, 2013. 
[20] M. Jaax, S. Wolff, B. Laegel, H. Fouckhardt, Optical and THz Galois diffusers, J. Eur. Opt. Soc. Rap. Public. 8 (2013) 13020-1-5.

[21] H.C. van de Hulst, Light Scattering by Small Particles, Dover Publications Inc., New York, 1981.

[22] A.A. Kokhanovsky, Optics of Light Scattering Media: Problems and Solutions, John Wiley \& Sons Ltd., Chichester, U.K., 1999.

[23] G. Mie, Beiträge zur Optik trüber Medien, speziell kolloidaler Metallösungen, Ann. Phys. 25 (1908) 377-445.

[24] A. Ehringhaus, H. Rose, Über die Abhängigkeit der relativen Dispersion der Doppelbrechung vom Atomgewicht, Z. Kristallogr. 58 (1923) 460-477.

[25] K.-H. Klaska, J.C. Eck, D. Pohl, New investigation of willemite, Acta Cryst. B 34, (1978) 3324-3325.

[26] A.B. Peck, A new glass stone: $\mathrm{Na}_{2} \mathrm{O} \cdot 3 \mathrm{CaO} \cdot 6 \mathrm{SiO}_{2}$, J. Am. Ceram. Soc. 9 (1926) 351-353.

[27] N.P. Bansal, R.H. Doremus, Handbook of Glass Properties, Academic Press, Orlando, Florida, 1986.

[28] F.H. Norton, The control of crystalline glazes, J. Am. Ceram. Soc. 20 (1937) 217224.

[29] E.O. Brigham, The Fast Fourier Transform and its Applications. Prentice-Hall, Englewood Cliffs, N.J., 1988.

[30] F.A. Jenkins, H.E. White, Fundamentals of Optics, fourth ed., McGraw-Hill, New York, 1976.

[31] M. Born, E. Wolf, Principles of Optics, seventh ed., Cambridge University Press, Cambridge, U.K., 2005.

[32] J.W. Goodman, Introduction to Fourier Optics, third ed.; Roberts and Company Publishers: Englewood, Colorado, 2005.

[33] P.W. McMillan, Glass-Ceramics, second ed., Academic Press, London, 1979.

[34] J. Zarzycki, Glasses and the Vitreous State, Cambridge University Press, Cambridge, U.K., 1991, pp. 161-166.

[35] C. Sun, C. Kuan, F.J. Kao, Y.M. Wang, J.C. Chen, C.C. Chang, P. Shen, On the nucleation, growth and impingement of plate-like $\alpha-\mathrm{Zn}_{2} \mathrm{SiO}_{4}$ spherulites in glaze layer: a confocal and electron microscopic study, Mater. Sci. Engng. A 379 (2004) 327-333.

[36] W.D. Li, J.Z. Li, Z.Q. Deng, J. Wu and J.K. Guo, Study on Ru ware glaze of the Northern Song dynasty: One of the earliest crystalline-phase separated glazes in ancient China, Ceramics International 31 (2005) 487-494.

[37] MiePlot 4.5, http://www.philiplaven.com/mieplot.htm. Downloaded August 2015. 


\section{Figure captions}

Fig. 1 Schematic diagram of the experimental arrangement used for initial optical experiments.

Fig. 2. (a) Schematic diagram and (b) actual experimental arrangement used for angular intensity measurements.

Fig. 3. A low magnification photograph of spherulites in a crystalline glaze

Fig. 4. (a) Detail of a polarised light micrograph taken at the edge of a spherulite, taken between crossed polars and a sensitive tint. (b) Diagram showing the extinction directions for the analyser (A) and polariser (P) respectively. The slow direction of the sensitive tint is also shown. Copious secondary nucleation has occurred around the primary willemite needles evident on the right-hand side of (a), resulting in smaller willemite spherulites some $150 \mu \mathrm{m}$ in size on the left-hand side of the micrograph. The willemite needles cannot be resolved optically in these secondary spherulites. The optically isotropic glass matrix with the colour of the sensitive tint is to the left of these secondary willemite spherulites.

Fig. 5. (a) Secondary electron SEM micrograph showing branching detail within a spherulite, and (b) secondary electron SEM micrograph showing detail within the main body of a spherulite and its surrounding periphery region. In both micrographs the mean growth direction is in the vertical direction. The contrast in this microscopy mode highlights the zinc-containing willemite crystals as long, thin, white branched needles on a darker grey background of silica-rich glass of a lower mean atomic number than the willemite crystals.

Fig. 6. (a) TEM micrograph of willemite crystals at the edge of a spherulite with the electron beam parallel to the [ $\overline{1} 2 \overline{1} 0$ ] willemite direction; (b) TEM micrograph showing detail within small spherulite crystals within the periphery region. The electron beam is parallel to the [10 10 0] willemite direction; (c) Further detail of the branching behaviour of willemite crystals. The electron beam direction was parallel to the [ $\overline{1} 101]$ willemite direction; (d) TEM micrograph of the glass region adjacent to the spherulites showing clear evidence of phase separation on a scale of $100 \mathrm{~nm}$.

Fig 7. An example of a diffusion pattern from a spherulite produced on the semitransparent hemispherical screen in Fig. 1.

Fig. 8. (a)-(c) Examples of three different regions of willemite needles images in a transmitted light polarising microscope with the extinction directions vertical and horizontal, together with (d)-(f) FFT images from these three regions and (g)-(i) the results of optical diffusion experiments from these three regions.

Fig. 9. (a), (c) Angular light intensity measurements for the corresponding diffusion patterns shown in (b) and (d) respectively. 
Fig. 10. (a)-(b) White light beam passing through various parts of the willemite crystal sample (shown in insets) producing different optical diffusion patterns. (c) Angular intensity distribution plot across the long (vertical) and short (horizontal) axes of the diffusion pattern shown in (a). A zoomed-in view of the boxed area in (c) is shown in (d). (e) Optical transmission through willemite crystals. The white scale bars in the insets in (a), (b) and (e) each represent $200 \mu \mathrm{m}$.

Fig. 11. Calculated intensity from the full-form Mie solution for non-absorbing spheres as a function of scattering angle for a particle with refractive index of 1.699 embedded in a medium with refractive index of 1.52 onto which light with a wavelength of $633 \mathrm{~nm}$ is shone: (a) particle radius of $230 \mathrm{~nm}$, (b) particle radius of $1300 \mathrm{~nm}$. 


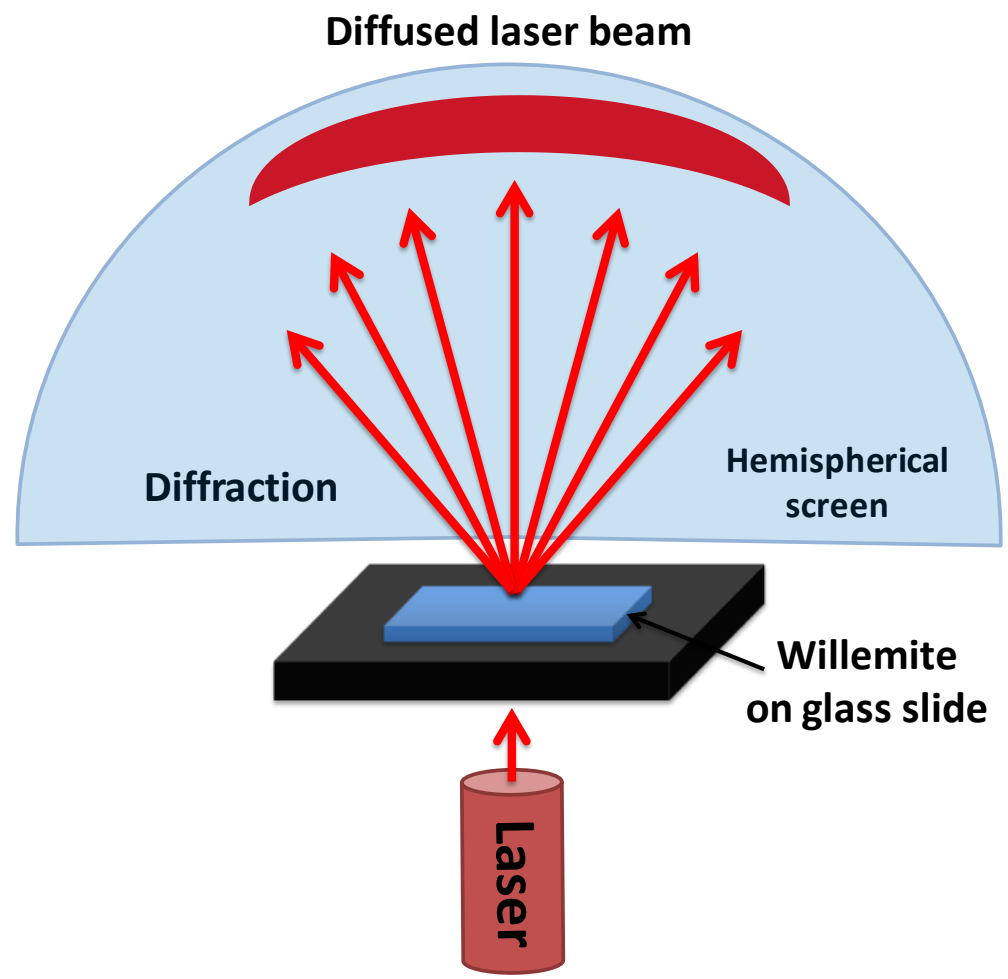

Fig. 1. Schematic diagram of the experimental arrangement used for initial optical experiments.
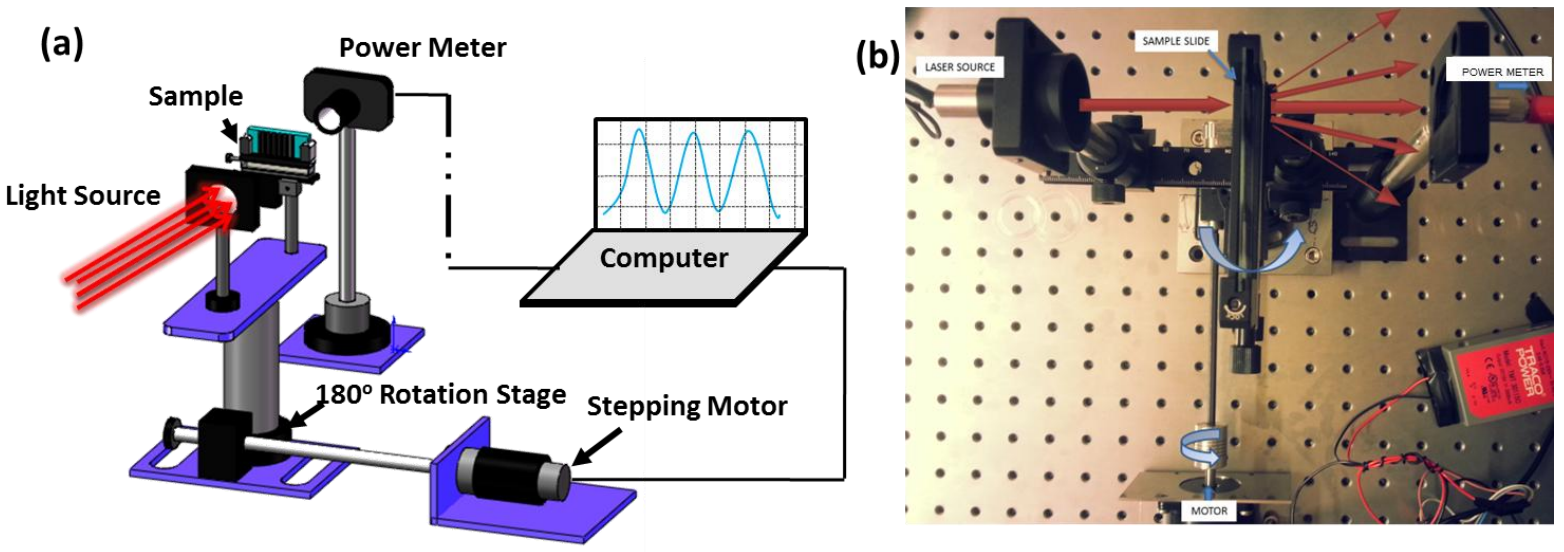

Fig. 2. (a) Schematic diagram and (b) actual experimental arrangement used for angular intensity measurements. 


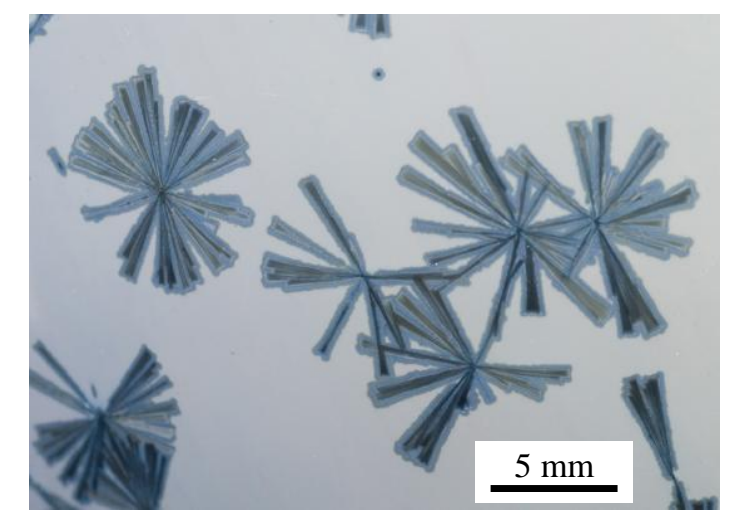

Fig. 3. A low magnification photograph of spherulites in a crystalline glaze.

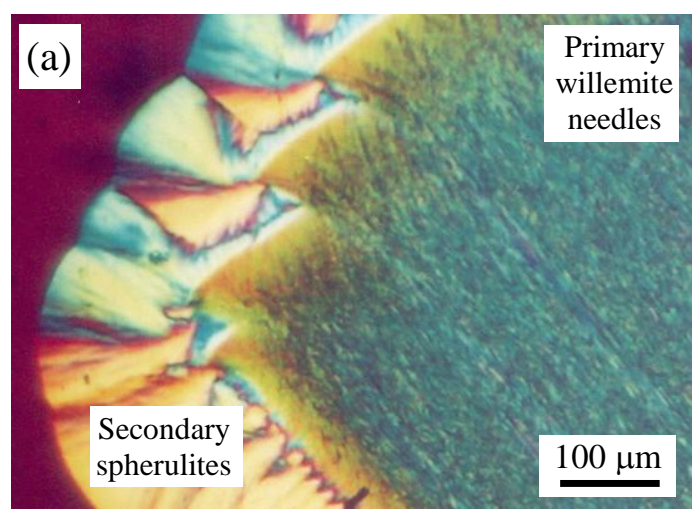

(b)

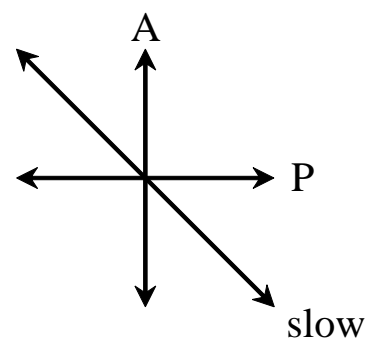

Fig. 4. (a) Detail of a polarised light micrograph taken at the edge of a spherulite, taken between crossed polars and a sensitive tint. (b) Diagram showing the extinction directions for the analyser $(\mathrm{A})$ and polariser $(\mathrm{P})$ respectively. The slow direction of the sensitive tint is also shown. Copious secondary nucleation has occurred around the primary willemite needles evident on the right-hand side of (a), resulting in smaller willemite spherulites some $150 \mu \mathrm{m}$ in size on the left-hand side of the micrograph. The willemite needles cannot be resolved optically in these secondary spherulites. The optically isotropic glass matrix with the colour of the sensitive tint is to the left of these secondary willemite spherulites. 


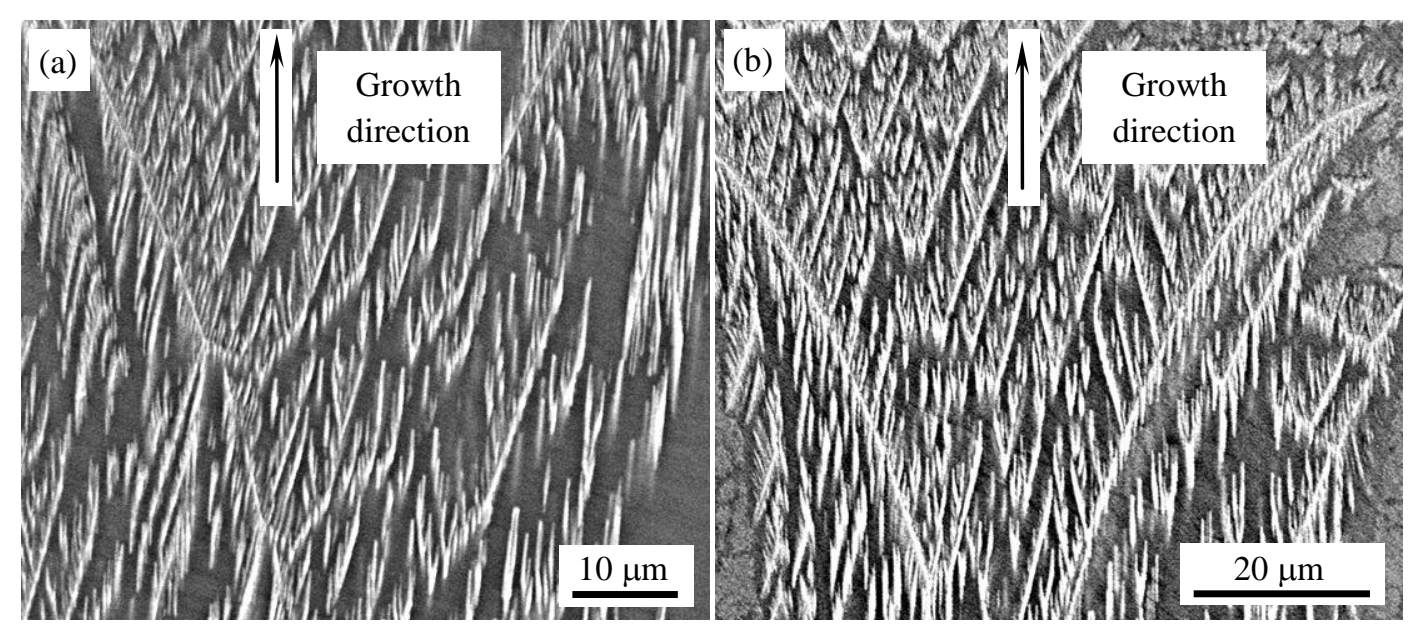

Fig. 5. (a) Secondary electron SEM micrograph showing branching detail within a spherulite, and (b) secondary electron SEM micrograph showing detail within the main body of a spherulite and its surrounding periphery region. In both micrographs the mean growth direction is in the vertical direction. The contrast in this microscopy mode highlights the zinc-containing willemite crystals as long, thin, white branched needles on a darker grey background of silica-rich glass of a lower mean atomic number than the willemite crystals. 

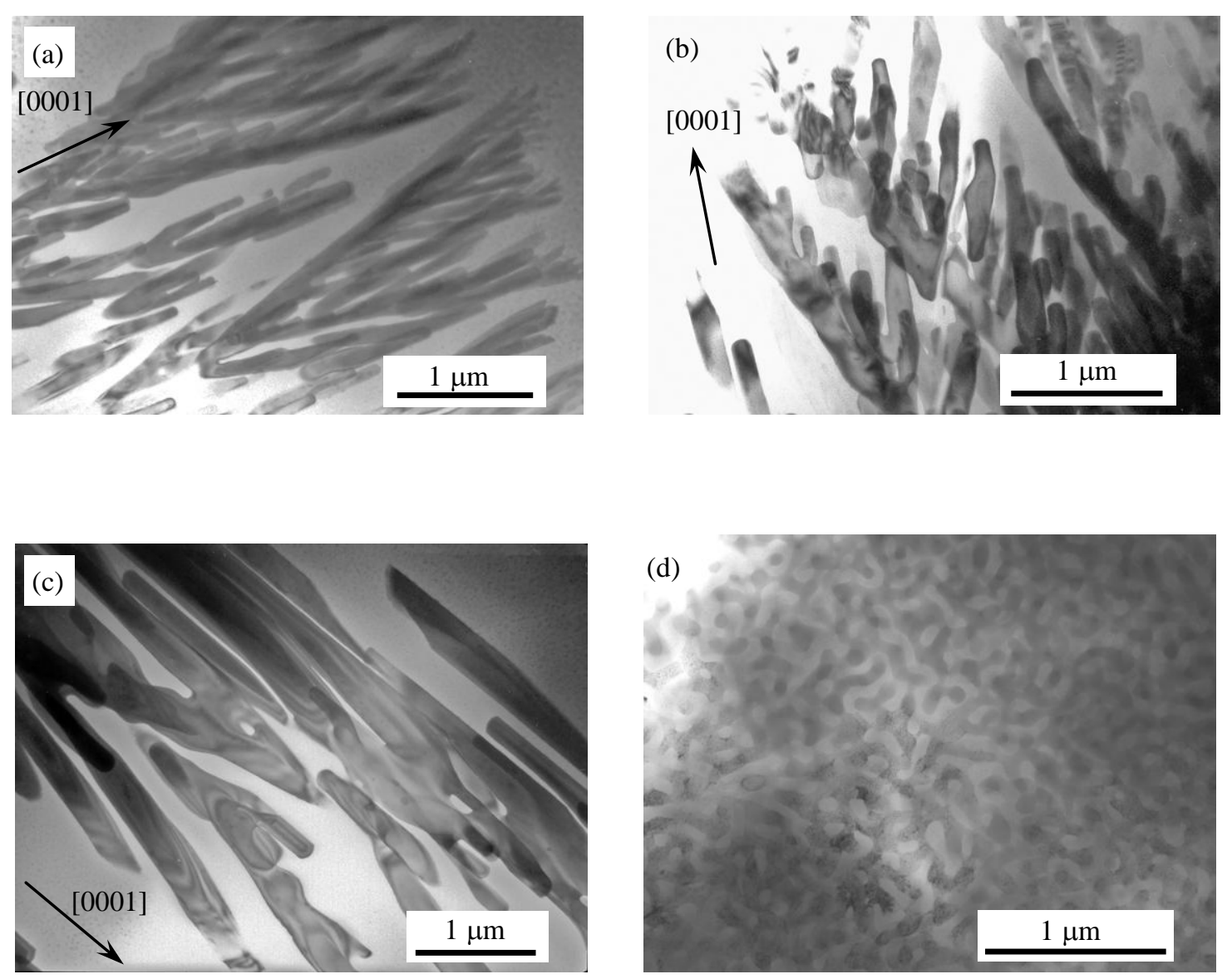

(d)

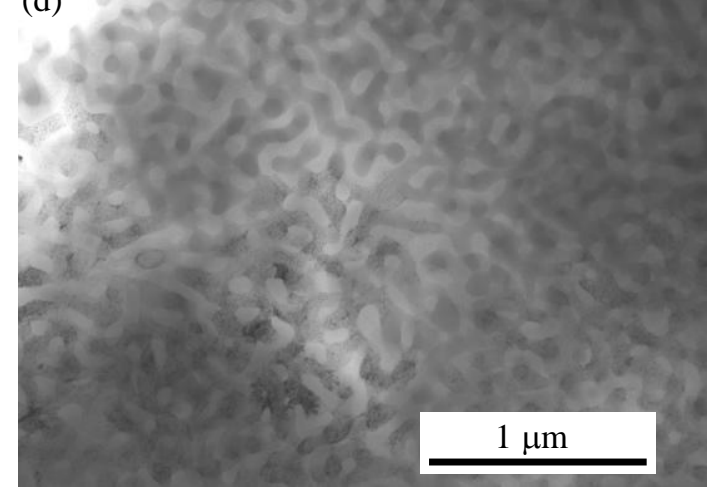

Fig. 6. (a) TEM micrograph of willemite crystals at the edge of a spherulite with the electron beam parallel to the [ $\overline{1} 2 \overline{1} 0$ ] willemite direction; (b) TEM micrograph showing detail within small spherulite crystals within the periphery region. The electron beam is parallel to the $[10 \overline{1} 0]$ willemite direction; (c) Further detail of the branching behaviour of willemite crystals. The electron beam direction was parallel to the [ $\overline{1} 101]$ willemite direction; (d) TEM micrograph of the glass region adjacent to the spherulites showing clear evidence of phase separation on a scale of $100 \mathrm{~nm}$. 


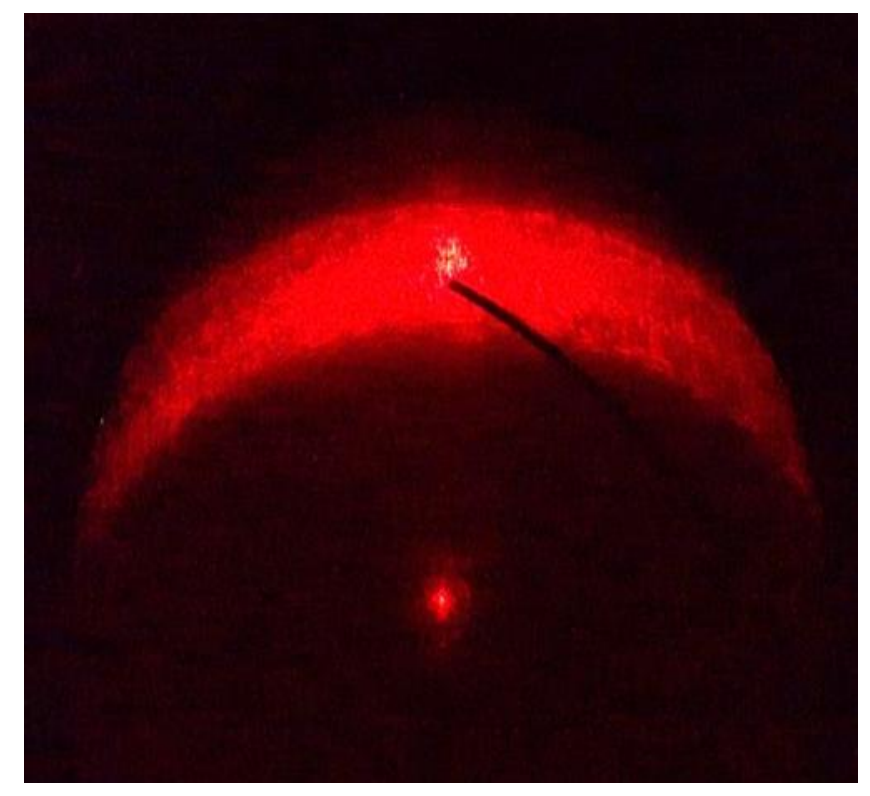

Fig. 7. An example of a diffusion pattern from a spherulite produced on the semitransparent hemispherical screen in Fig. 1. 

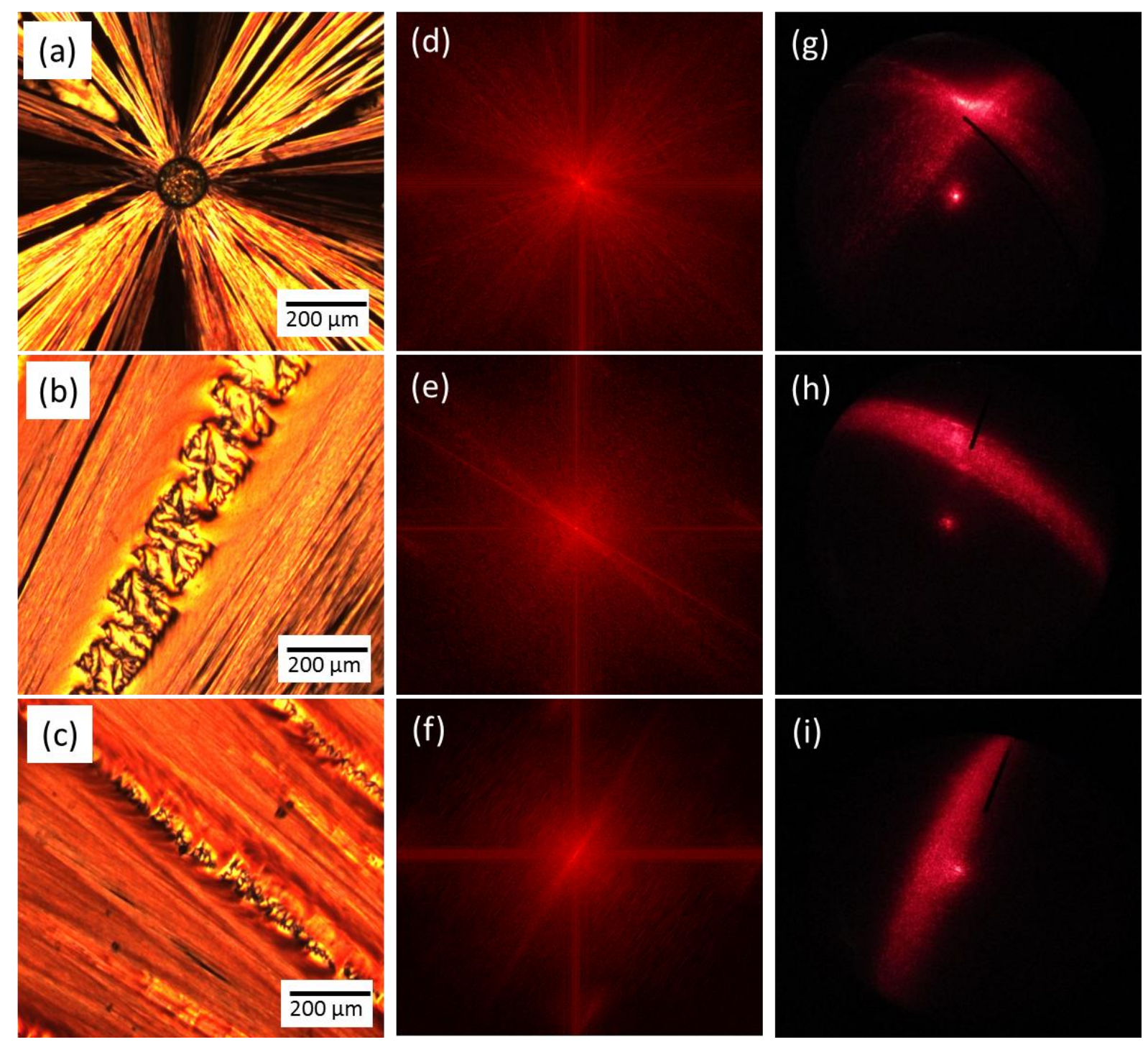

Fig. 8. (a)-(c) Examples of three different regions of willemite needles images in a transmitted light polarising microscope with the extinction directions vertical and horizontal, together with (d)-(f) FFT images from these three regions and (g)-(i) the results of optical diffusion experiments from these three regions. 
(a)

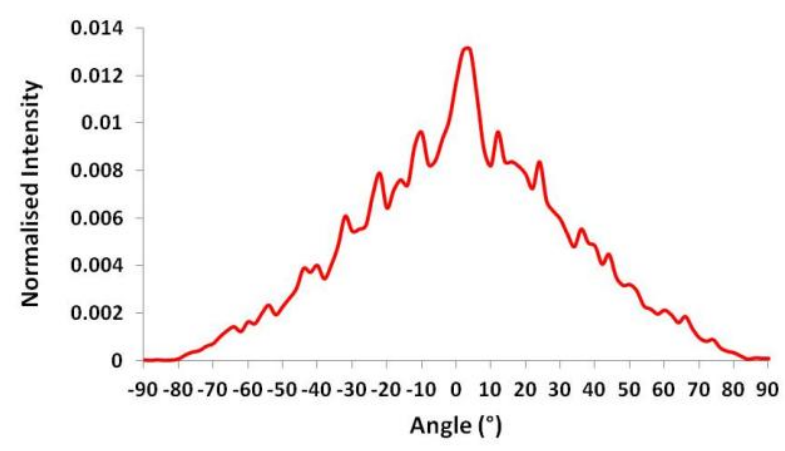

(c)

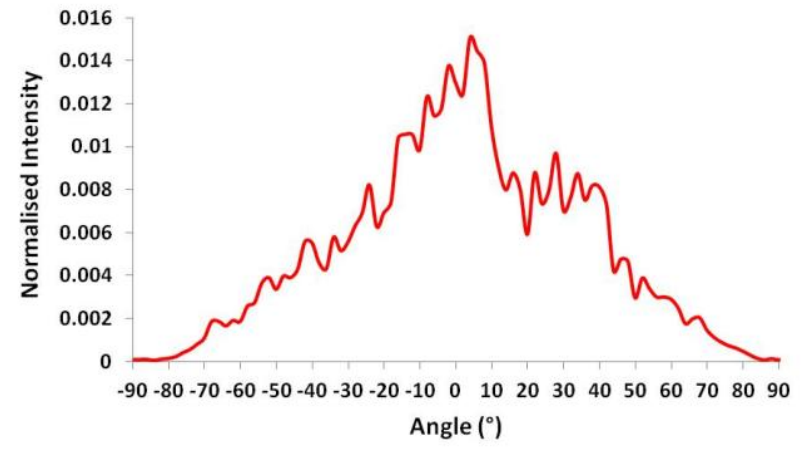

\section{(b)}

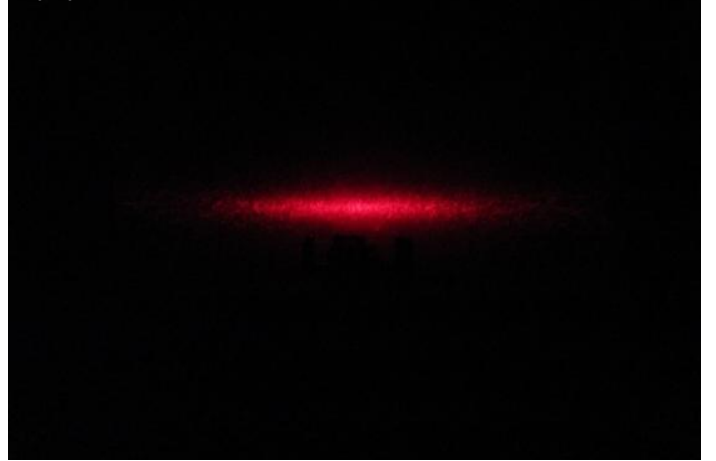

(d)

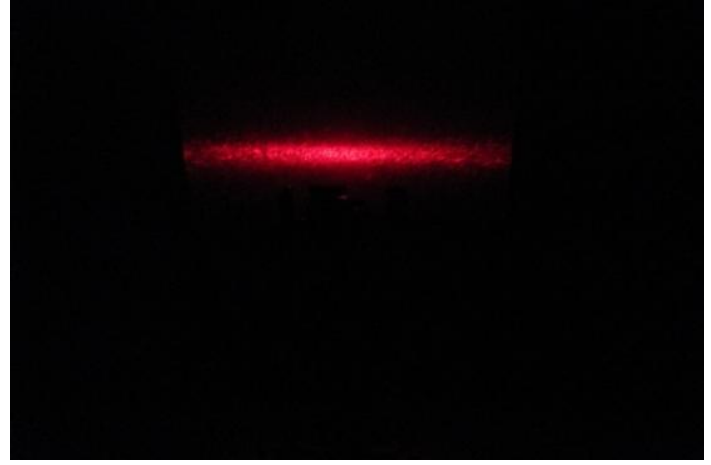

Fig. 9. (a), (c) Angular light intensity measurements for the corresponding diffusion patterns shown in (b) and (d) respectively. 

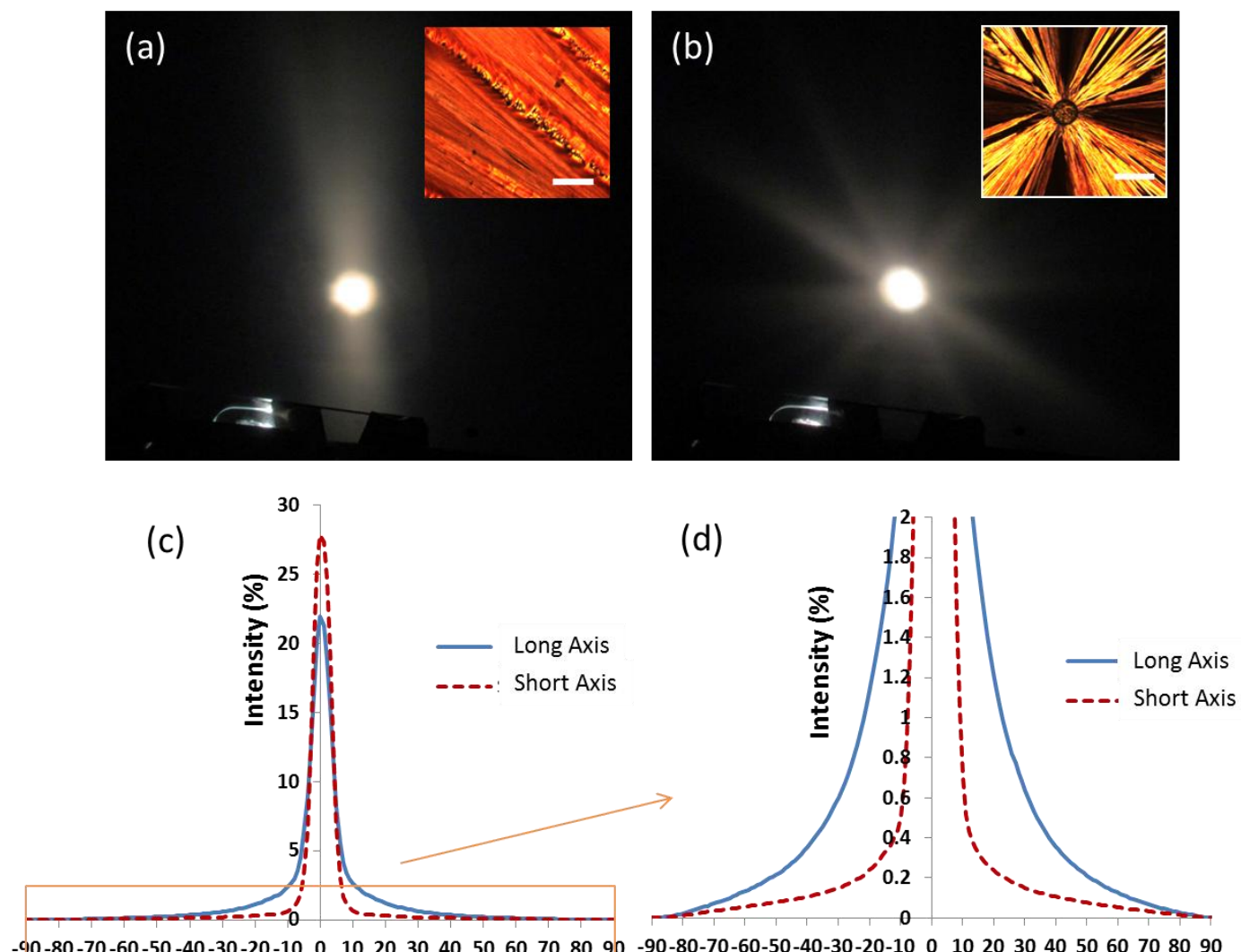

$-90-80-70-60-50-40-30-20-100102030405060708090-90-80-70-60-50-40-30-20-100102030405060708090$ Angle (deg) Angle (deg)

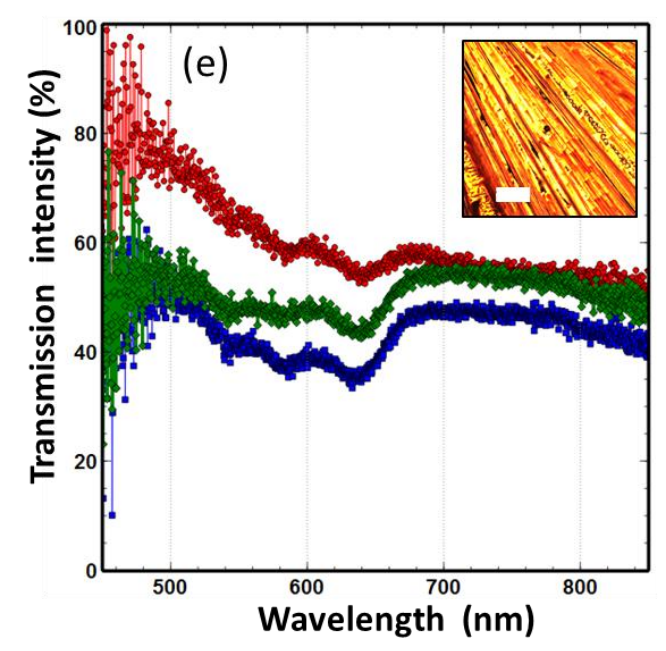

Fig. 10. (a)-(b) White light beam passing through various parts of the willemite crystal sample (shown in insets) producing different optical diffusion patterns. (c) Angular intensity distribution plot across the long (vertical) and short (horizontal) axes of the diffusion pattern shown in (a). A zoomed-in view of the boxed area in (c) is shown in (d). (e) Optical transmission through willemite crystals. The white scale bars in the insets in (a), (b) and (e) each represent $200 \mu \mathrm{m}$. 


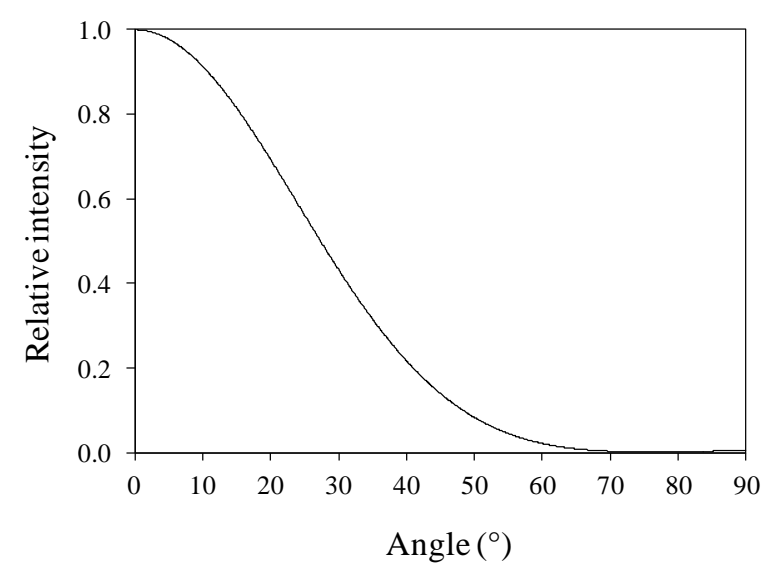

(a)

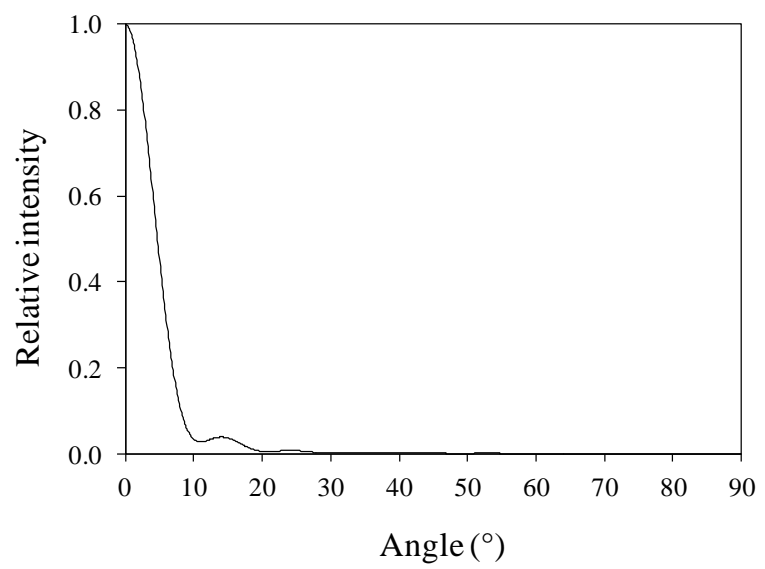

(b)

Fig. 11. Calculated intensity from the full-form Mie solution for non-absorbing spheres as a function of scattering angle for a particle with refractive index of 1.699 embedded in a medium with refractive index of 1.52 onto which light with a wavelength of $633 \mathrm{~nm}$ is shone: (a) particle radius of $230 \mathrm{~nm}$, (b) particle radius of $1300 \mathrm{~nm}$. 


\section{Highlights}

- Willemite spherulites embedded in glass act as optical diffusers of visible light

- The scale of the microstructure of willemite spherulites causes the effect

- The phenomenon is shown to be in essence a form of Mie scattering

- Optical diffusers based on willemite have potential for high temperature use 